\title{
Inter-Vehicle Communication Using Li-Fi Technology
}

\author{
Muntazir Mohd Tali ${ }^{1}$, Aarti Patil ${ }^{2}$, Sushmita Chavan ${ }^{3}$, Prof. Shailesh Jadhav ${ }^{4}$ \\ UG Student, Electronics and Telecommunication, Dhole Patil College of Engineering, Pune, India ${ }^{1,2,3}$ \\ Assistant Professor, Electronics and Telecommunication, Dhole Patil College of Engineering, Pune, India ${ }^{4}$
}

\begin{abstract}
Communication between vehicles, we present the blueprint and outcome of a small-scale mock-up using light fidelity (Li-Fi) technology, a new technology that was founded in the last January 2010 to January 2012, which still needs more organized inquiry on its sustainability for communication between vehicles. Inter-vehicle communication is an effective method with productive results that we have used in order to communicate between two vehicles and maintain safe distance between vehicles to prevent accedents. In Li-Fi technology, for communication between two vehicle data is transmitted using bulb and at receiving end we use photo detector to receive the data. In this technology there is no protocol used so it reduce the complexity. The aim of the paper is to design a module for communication between vehicles and to maintain safe distance between vehicles to prevent accedents.
\end{abstract}

Keywords: Inter-vehicle communication, Visible Light Communication, Light Emitting Diode, Photodiode.

\section{INTRODUCTION}

In this paper we are presenting the communication between vehicles using light as medium and maintain safe distance between vehicles to avoid accedents.

Insimple terms, Li-Fi can be thought of as a light-based $\mathrm{Wi}-\mathrm{Fi}$. That is, it uses light alternative for radio waves to transmit data. And instead of Wi-Fi system, Li-Fi would use LED lamps that can be used to light a room as well as transmit data.And at receiving end we use photodiode. Since simple light bulbs are used to transmit the data hence provides more security.

This system is proposed for communication between vehicles and maintains safe distance between vehicles. The line of sight between vehicles should be clear.

\section{OBJECTIVES}

It is used to give different or add-on to existing wireless technology .To reestablish high speed connection.Li-FI is used because it is fast and secured version of Wi-Fi and is very cheap as compare to $\mathrm{Wi}-\mathrm{Fi}$.

\section{III.WITHOUT WI-FI OR GPS}

Husain Fidvi [4] has proposed Vehicle to Vehicle communication that does not need GPS or Wi-fi connectivity.It was proposed to use programmable interface controller (PIC) sonar which sends 40kHZ sound pulse that is not detected by human ears. The echo from the front vehicle is deteced by the microcontroller.The distance between the vehicle is calculated by total time taken by the sound signal to come back. This technology is shown in fig 1

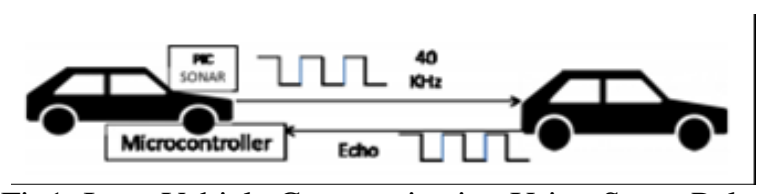

Fig1: Inter-Vehicle Communication Using Sonar Pulse

Various research works have been done in literature for vehicles to vehicles communication using an advantage of light. As the frequency spectrum of light is large, it is favourable to be adopted in a limited-range wireless communication. In this project a system is developed in order to build a cost effective yet inexpensive mechanism for Inter-vehiclescommunication through the use of a visual wireless communication medium, which is light. In fact, complex wireless networks and protocols can be eliminated using LED.

\section{IV.METHODOLOGY}

Communication between Vehicles and calculation of distance between vehicles is achieved by using Spread Spectrum Technique and ultrasonic sensor. In this, a vehicle (Vehicle-A) which is having ultrasonic sensor gives the information about distance between target vehicle (Vehicle-B). However, in this system, communication between only two vehicles is done, we can also design number of target Communication and ranging technologies for communication between vehicles by Using Spread Spectrum Technique.In this project we can send messages to the receiving vehicle one by one depending on condition.We are also using speakers for voice output and LCD to display message on both transmitter and receiver side.We are also using ultrasonic senser to keep safe distance between vehicles to avoid 
accidents. If the distance between vehicles is less then that of minimum distance then buzer will get turned on.

\section{PROPOSED SYSTEM}

The propose plan of action for our project is inter-vehicles communication using optical wireless model having high data rates (in the range of $\mathrm{MHz}$ to $\mathrm{GHz}$ ) and transmission distances is near about $1 \mathrm{~m}$. For transmission of information from one device to another device required LED. In this system at the transmitter section input data given using switching control system. According to the data, the microcontroller generates a stream of $1 \mathrm{~s}$ and $0 \mathrm{~s}$ thereby translate the data in binary. The output of this controller is given to the LEDs which is connected to transmitter side. Which turn $\mathrm{ON}$ and $\mathrm{OFF}$ at very high speeds. This ON-OFF regulate the transmits of data through light. LED is the selected for light source since it consumes very less power when compared to fluorescent lamp or a light bulb. It consumes very less power then that of conventional method for lightning that is one-tenth of the power required.Also, the lifespan of a typical LED bulb is several tens of thousands of hours. LEDs also have quick switching with good visibility. Thus, LEDs are the best choice for transmitting the information. On receiver section, photo diode is used such as silicon photo detector. The photo detector decodes the incoming received information based on the sequence of $1 \mathrm{~s}$ and $0 \mathrm{~s}$. Then the decoded signal is given to output device such as LCD display or speaker. Thus Li-Fi network is confirmed

\section{VI.SYSTEM ARCHITECTIRE}

According to the condition, user will give the input to the microcontroller using keypad switches.Microcontroller will convert the input into ASCII \& then the ASCII value is given to output pins of microcontoller on which $\mathrm{Li}-\mathrm{Fi}$ transmitter is connected.Li-Fi converts ASCII value into visible light spectrum. Now at the receiver side, Li-Fi receiver will receive the message sent by LED (continious 0 's and 1's) and decode the information and gives it to the output unit LED and speaker

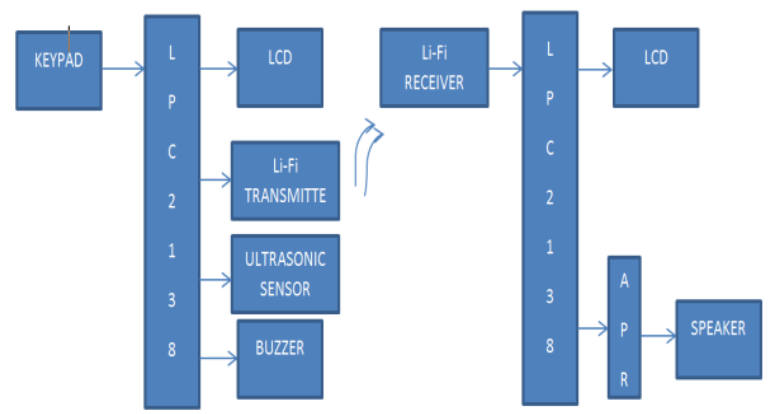

Fig. 2: Block Diagram of Inter-Vehicle Communication Using $\mathrm{Li}-\mathrm{Fi}$

The functionality and use of each block of the system is explained below
A. Li-Fi Transmitter

It receives the information from the microcontroller according to the condition and it converts the data to light signal and transmits to the receiver section. The transmitter part modulates the input signal with the required time period and transmits the data in the form of $1^{\text {ee }} \mathrm{s}$ and $0^{\text {ee }} \mathrm{s}$ using a LED bulb. Modulation of light can not be detected by human eyes making data secured.The sending of data has been made possible by the use of LED bulbs. These bulbs can be continously switched $\mathrm{ON}$ a OFF Very fast.Thus makes it possible to send data via light.

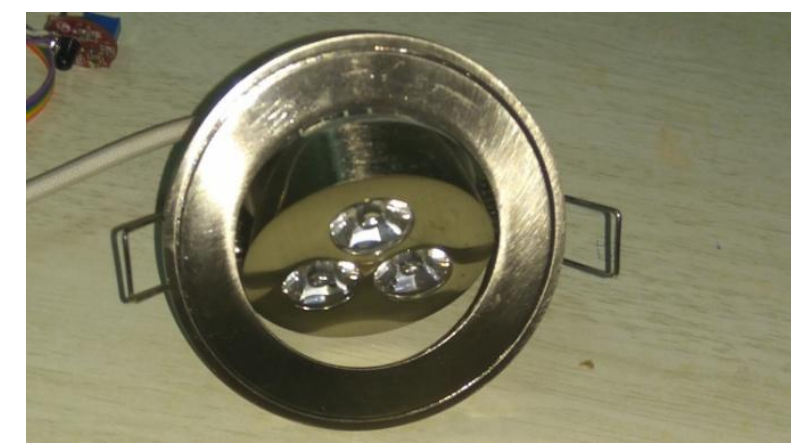

Fig 3: Li-Fi Transmitter.

\section{B. Ultrasonic Sensor}

Ultrasonic sensors are characterized by their reliability and outstanding versatility.Ultrasonic sensors can be used to solve even the most complex task involving object detection or level measurement with millimeter precision because their measuring method works reliably under almost all condition.It is based on measuring the properties of sound wave with frequency above the human audiable range.Calculation of distance is given as Distance $=$ Speed of sound $*$ Time taken $/ 2$ Speed of sound in air $344 \mathrm{~m} / \mathrm{s}$

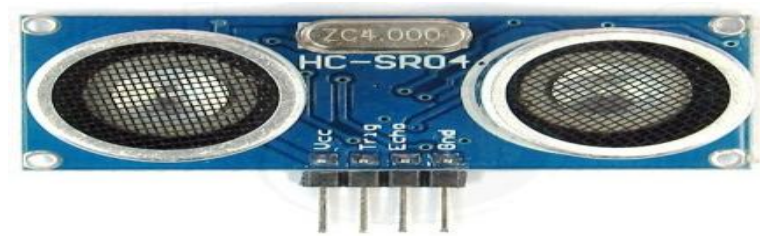

Fig 4: Ultrasonic Sensor.

\section{Li-Fi Receiver}

It receives the modulated signal send by $\mathrm{Li}-\mathrm{Fi}$ transmitter and demodulates the signal to recover the original signal.The receiver part detects these flashes using photodiode and then sends demodulated signal to microcontroller.

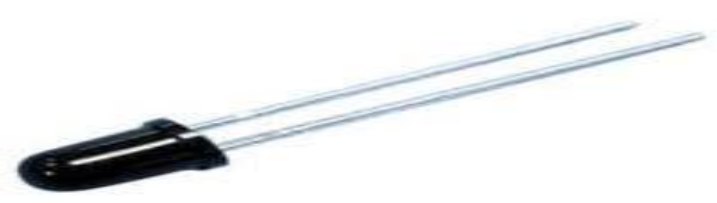

Fig 5: Photodiode. 


\section{APR Module}

ARP is a single chip voice recording and playback device for 20 to 30 seconds voice recording and playback.It is an ideal IC for automatic answering macchine.This IC has data storage capacity and requires no software and microcontroller.It provides high quality voice recording and palyback upto 30 seconds. This IC requires minimum components to create a voice recorder.The IC has nonvolatile flash memory technology with $100 \mathrm{k}$ recording cycles and 100 years message retention capacity. The LED glows when the IC records the voice obtained through the mic. The IC remain in the recording mode as long as RecL pin is grounded.By pressing the playback switch the play mode starts from the beginning of the message.The input section will be muted during playback.

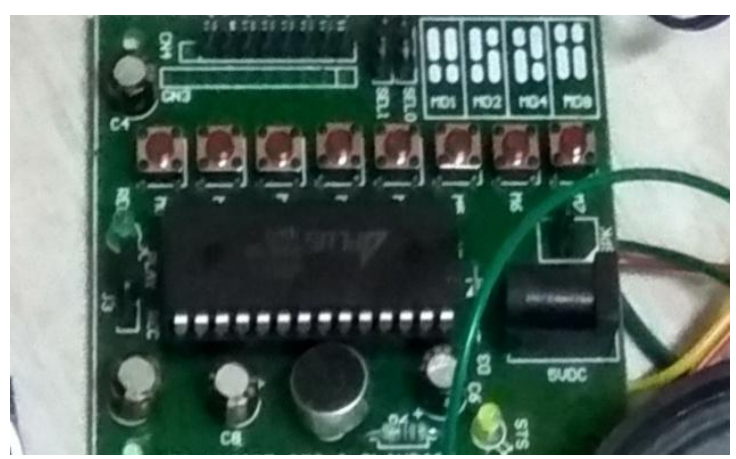

Fig 6: ARP Experimental Board

\section{RESULT}

Li-Fi Transmitter and Receiver module

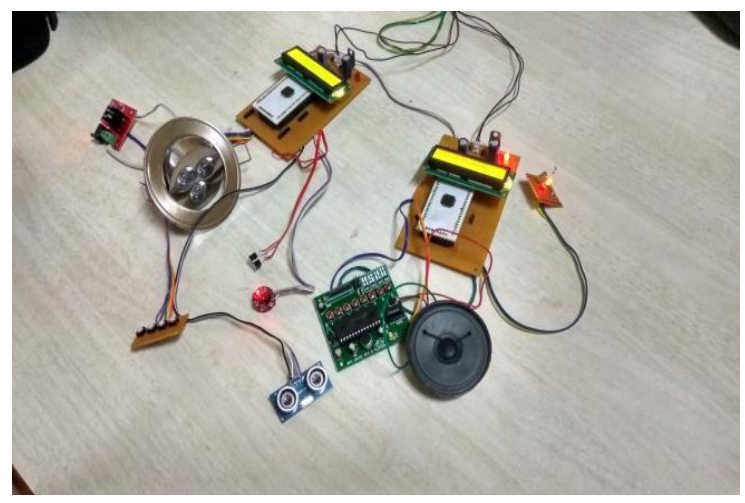

A. Information of Fuel Leakage

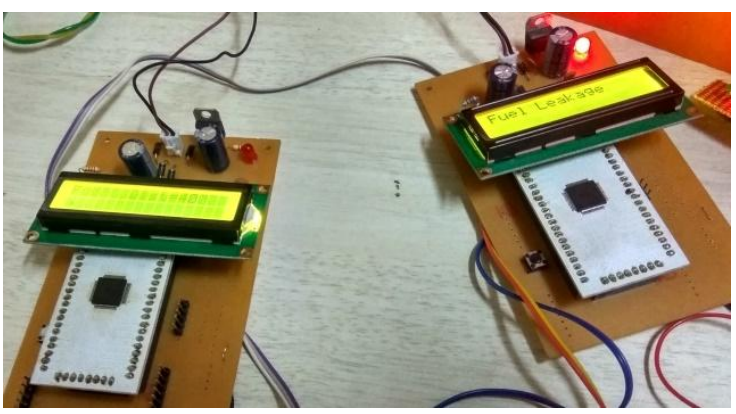

Fig 7: LCD Displaying Fuel Leakage.

\section{B. Information of Rash Driving}

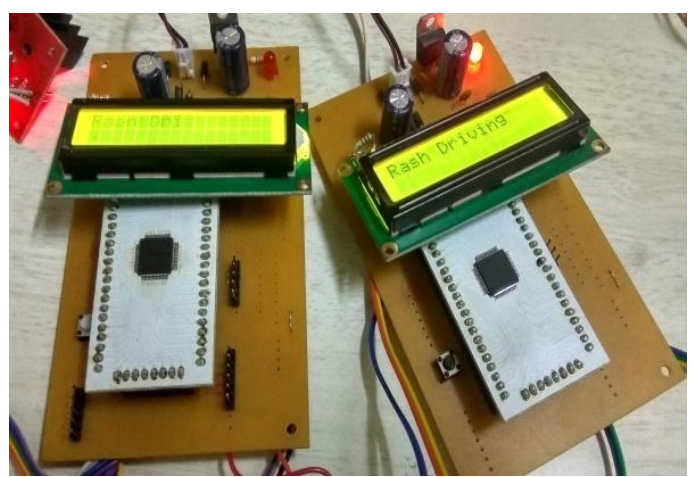

Fig 8: LCD Displaying Rash Driving

\section{CONCLUSION}

We have presented an Inter-Vehicle Communication system consisting of a Li-Fi transmitter and receiver that is targeted at communication between vehicles, Li-Fi can be used to communicate with the LED lights of the cars and number of accidents can be prevented. Li-fi is ideal for high density coverage in a restricted region.It is believed that the technology can yield a speed more than 10Gbps.It is the fastest and cheapest wireless communication systems which is suitable for communication.Li-Fi will make all our lives more technology driven in the near future.

\section{REFERENCES}

[1] S.Vicky,"Li-Fi The Best Alternative To Overcome The Existing Technologies" Vol. 2, Issue 2, pp: (208-212), Month: April-June 2014

[2] Shubham Chatterjee,"Scope and Challenges in Light Fidelity(LiFi) Technology in Wireless Data Communication "Issue 6, Volume 2 (June 2015)

[3] Shaista Tarannum,"Data Transmission Through Smart Illumination Via "Visible Light Communication Technology" Volume 4, Issue 2 (March-April, 2016), PP. 136-141

[4] N. M. Husain Fidvi, "Car to Car Communication System," source: car communication system

[5] M.Chinnadurai,"Smart LI-FI Based Car Parking System. "Volume:03,Issue 022016

[6] http://caledonianmercury.com/the-futures-bright-the-futures-li-fi

[7] http://www.lifi-centre.com/about-li-fi/applications 